\title{
The Impact of Blended Learning using the Ideas Box on the Motivation for Learning Among Non-formal Syrian Female Refugee Students in Jordan
}

\author{
https://doi.org/10.3991/ijim.v15i11.21961 \\ Asma'a S. Alkhatib ${ }^{(凶)}$, Suhair A. Jaradat \\ University of Jordan, Amman, Jorden \\ Khatib.asmaalyahoo.com
}

\begin{abstract}
The study aims to identify the impact of blended learning using the Ideas Box on the motivation toward learning among non-formal education female students in the Syrian refugee camps in Jordan. A semi-experimental approach was adopted; the sample of the study consisted of 30 female students enrolled in the Culture Promotion Program for Dropouts, second cycle, in the JOHUD Center for Social Support in January 2020. The experimental group consisted of 15 students who were taught the Islamic Pillars unit using the Ideas Box while the control group consisted of 15 students taught the same unit using the traditional way. Results show that there were no significant differences in mean scores of motivation between the two groups. This finding stresses the need for redesigning the current program used to teach students receiving the non-formal education to incorporate the Ideas Box into the curriculum.
\end{abstract}

Keywords - Blended learning, Ideas Box, motivation toward learning, non-formal education, Culture Promotion Program for Dropouts

\section{Introduction}

The Hashemite Kingdom of Jordan is a country with limited natural and financial resources but is also a country that receives a large number of refugees. At the same time, Jordan gives priority to education and tries hard to maintain a high quality of education that distinguishes it globally and preserves its Arab identity. This advantage would enable the small country to face the challenges imposed by the modern technology and information communication revolution as well as humanitarian crises.

The Jordanian education model is seen as a pioneering model in the Middle East and North Africa region although it has been in decline since 2016 [13]. This is due to the fact that the educational system has been facing great challenges in providing education to all children residing in Jordan, including refugees on its lands; the country is struggling to maintain the quality of education. In terms of access to education, the situation of Jordanian children is much better than that of Syria and other countries as Jordanian children have reached gender parity in Kindergarten II and the primary stage. As for the secondary stage, the total enrollment rate for Jordanian males is lower than that of 
females. This might be caused by poverty and the need for males to join the labor market as unskilled workers, which causes males to drop out of school at a higher rate than females. It may also reflect the lower quality of government schools as male teachers tend to be engaged in more than one job [12].

On the other hand, Syrians' acceptance of education is much lower than Jordanians at all levels of education; the percentage of Syrian refugees enrolled in basic education was about 37\% during the 2015-2016 academic year. However, the percentage of Syrian refugees enrolled in kindergarten and secondary education is much lower, reaching about $9 \%$ for kindergarten and $14 \%$ for secondary. It has been concluded from these ratios that Syrian girls are the most deprived of a secondary education. It is worth noting here that most Syrian students can go to public schools as is the case for Jordanians [12]. According to Care International and Plan International [6], 54\% of Syrians, $85 \%$ of Jordanians, and $80 \%$ of Iraqi children go to school. Among the age groups within the school and university stage, only $28 \%$ of Syrians, $58 \%$ of Jordanians, and $74 \%$ of Iraqis attend school or university.

As for those enrolled in school, the dropout rate is rising, especially for Syrian refugees where the dropout rate is $20 \%$ for those living in camps and $27 \%$ for refugees living in cities. The reasons lie in the inability to afford the costs and their belief in the insecurity of transportation from their own perspective. Moreover, the greatest difficulties that Syrian refugee students face in Jordan are harassment and discrimination as well as a lack of normalcy and coping strategies for different life situations [16].

Perhaps the most important component of reaching feasible solutions regarding problems of access to education, as well as school dropout, would be to invest in alternative and existing programs of the MOE. Such programs should be developed to activate the optimal investment of technology in its various forms, for its relevance to the goals and spirit of our age, besides its suitability for the target groups. The emergence of various forms of e-learning has helped in finding appropriate solutions to solve the problems of access to education and dropout, as they were commensurate with the needs of learners and their different characteristics as well as the nature of the available communication tools. These technological innovations have helped make the learner the focus of the educational process instead of the teacher. One such innovation is blended learning, which is the use of varied technology to deliver information to the learner with the least time and effort [3].

Blended learning is considered as a method for designing and implementing educational curricula, combining the characteristics of both face-to-face and e-learning through different technologies. It does not replace the individual methods but rather creates a new learning experience for the learners. People who are developing blended learning seek to increase the efficiency of what individual learners do on their own (e.g., discussions, group presentations, or reflections) by mixing it with what they do on the Internet. In this way, the effectiveness of the traditional study hours in the classroom will get boosted, with some equal mix of face-to-face work and work on the Internet. In other words, it is not devoid of the humanitarian aspect [1].

The Ideas Box [9] — a pop-up multimedia center and portable library-is considered one of the important technological tools used in many places and contexts to support the learning process due to the variety of tools in the different boxes. This contributes 
to improving the learning process as well as encouraging students to self-learn due to the ease of use of the tools and its availability at any time they prefer.

The learning process needs learning to be an effective process, and that is why the learner is supposed to have a drive, desire, and passion to learn. This is because motivation represents a special condition for the learner that drives and directs their attention and focus to the learning process. It also creates activeness and vigorousness in order to continue the learning process until its goal is achieved. The responsibility for stimulating students' motivation to learn rests on both home and school [14]. The use of modern technology and its provision for students represent an input that can have a positive impact on increasing students' motivation to learn, especially those who drop out.

In light of previous perspectives, this study attempts to examine the effect of blended learning using the Ideas Box on the motivation toward learning among non-formal education female students in the context of the Syrian refugee crisis in the host communities in Jordan.

\section{$2 \quad$ Study Problem and Questions}

In general, there is a lack of educational opportunities as the Jordanian education system faces many challenges (e.g., demographic changes and the influx of refugees) that cause unequal educational outcomes. Classrooms are overcrowded, and fewer school hours are sometimes given due to the double-shift system that led $19 \%$ of Syrian refugees and $29 \%$ of Iraqi refugees to express their need for additional assistance with education. Moreover, there are other obstacles that basic and higher education face: lack of community and family participation, lack of communication and coordination under a centralized system, and need for further training of teachers. There is a lack of quality and relevance of content that makes it appropriate to the current needs of both society and the labor market [6], which directly influences the motivation of students toward learning.

As a result of these circumstances, many students have dropped out of school and are unable to return to regular education because of the MOE policy of a 3-year time limit on return after dropout. Some join the MOE dropout centers, but many do not return because they need to work to support their families. This makes them unable to commit to working for long periods or permanently in centers that promote the culture of dropouts. In other cases, girls marry at an early age and are deprived of educational opportunities; often they are not allowed to leave home for long periods. Some students drop out as a result of bullying classmates and the poor quality of education in schools, especially the presence of large numbers in the classroom. All of these factors affect the quality of education and do not give all students their equal rights in class, generating more dropout students who do not possess the basic skills of reading and writing and have no motivation to learn.

The two authors have worked with the Jordanian Hashemite Fund for Human Development (JOHUD) Center for Social Support, which is one of the centers in the Kingdom that deals with dropouts; it uses the Ideas Box to develop students' motivation 
toward learning. We noted that the Center used this tool in a limited and unstructured way, based on personal judgments that may or may not be correct. We also noted that students became bored while carrying out the tasks required of them in the usual way and the redundancy of exercises and activities in the curriculum taught at the Center; both of which limited their motivation toward learning. These circumstances are reinforced by the parents' lack of interest in education in general and not considering it a priority. Another factor is lack of awareness among facilitators of the need to use the available modern technology in a systematic and deliberate manner in the educational process.

Despite the existence of the Ideas Box at the Center for about 5 years, no evaluation or study has been conducted to determine whether there is an actual impact of the Ideas Box on achievement or any other variable. Given that the Ideas Box possesses attractive characteristics related to the use of multimedia (e.g., sound, pictures, and colors), the artistic design of the box, and the tools inside the boxes that work to develop thinking, this study was conducted to determine the effects of the Ideas Box on motivation.

In general, studies that have investigated the effect of blended learning based on the Ideas Box on motivation toward learning are rare. To our knowledge, the current study may be one of the first at the Arab world's level that combines the development of motivation toward learning with the study of the Ideas Box. This study was conducted upon the recommendation of the Center. It is very important to note that in our literature search we did not find any Arabic study related to the target group in this study (i.e., dropouts) that dealt with any of the above or other variables, which indicates the weak interest in this target group. Therefore, this study may help individuals or organizations interested in this category of society.

This study attempts to design a teaching program to teach the first unit, the Pillars of Islam, in the integrative approach and the second cycle using the Ideas Box. The study also aims at measuring the impact on developing the motivation toward learning among female students of informal education in the context of the Syrian refugee crisis in host communities in Jordan. The study tried to answer the following question:

- Are there statistically significant differences $(0.05 \geq \alpha)$ between the mean scores of the study subjects in the motivation of the students toward learning due to the method of teaching using blended learning based on the Ideas Box?

\section{Purpose of the Study}

This study's objective is to determine the effects of blended learning using the Ideas Box on motivation toward learning among non-formal education female students. The students' ages ranged between 14-18 years at the time of the study. The students were located at the JOHUD Center for Social Support in Amman - Marka, Jordan. 


\subsection{Importance of the study}

The importance of the current study stems from the necessity to update and develop teaching methods and styles, and the interest of educational institutions to employ technological developments in educational learning situations. Based on the findings of research studies on the importance of technology in education, the significance of this study at the practical level comes from the need to improve and develop the strategies followed in education in general and, more specifically, in non-formal education programs such as the program to enhance the learning of dropout students by making use of available technology in developing their motivation to learn. It also aims at helping learners who have dropped out of school to overcome the difficulties they face in learning concepts and their applications in life situations. It may qualify students to be capable of renewal and modernization to keep pace with global challenges while deepening the idea of preserving the cultural authenticity of society, which leads to the prosperity, growth, and development of society. This study may help direct the Center's facilitators to adopt blended learning using the Ideas Box in developing their design abilities and achieving their concept of professional growth by searching for new teaching methods characterized by creativity and modernity.

This study can contribute to clarifying the role of parents who support, direct, and enhance the learning of their children and develop their motivation toward learning. This can be achieved by providing counseling sessions for them by the Center facilitators to make them aware of the importance of encouraging their children to continue learning and investing in technology for learning. This would be positively reflected in directing children to be well prepared for this future learning, which helps them in working with the Center to complete their educational journey, regardless of the challenges they face and the duration of their education interruption.

Additionally, there is an urgent need to know the value added by the Ideas Box versus the money invested, which includes cost of purchase, maintenance, and operation. This will benefit the JOHUD by expanding access to additional funds to support its other centers spread across the Kingdom's governorates. As for decision-makers, the study will benefit them by circulating its results to the MOE, international organizations, and decision-makers in education and educational development to activate the efficient and effective use of technology. It will also help focus on motivation toward learning and investing technology in the MOE's Culture Promotion Program for Dropouts in particular and perhaps the reassessment of the educational design of that program.

Finally, this study may help enrich the Arab library with its results on the impact of blended learning using the Ideas Box on the motivation toward learning among nonformal education female students, in the context of the Syrian refugee crisis in the host communities in Jordan. 


\subsection{Terminology of Study}

Blended Learning. Ref. [8] defined blended learning as "learning that is facilitated by the effective combination of different modes of delivery, models of teaching and styles of learning, and founded on transparent communication amongst all parties involved with a course." (p. 12). We define it procedurally as follows: Learning that combines regular face-to-face learning and e-learning using the Ideas Box, through the use of various technological tools and means that are contained in the box, and stimulates the students' attention in order to motivate them to learn, hence improving their educational learning process outcomes.

Ideas Box. Ideas Box is defined by Ref. [9], the non-profit organization that created and operates it, as a multimedia mobile center and a learning hub that provides educational and cultural resources to communities in need, including refugees and displaced persons in camps around the world, and in disadvantaged communities in developed countries. We define it procedurally as a technology center consisting of four basic boxes, each of which has specific tasks and functions based on the technological equipment it contains. All of these boxes are used to support the education process under the supervision of the facilitator.

Blended Learning using the Ideas Box. We define this combination of the previous two terms procedurally as learning that combines regular learning in the classroom and e-learning using the Ideas Box, which consists of four boxes with different goals that support the learning process.

Motivation toward Learning. Motivation toward learning is the set of internal and external conditions that drive the individual in order to restore the balance that has been disturbed. Motivation, in this sense, indicates a tendency to reach a specific goal; this goal may be to satisfy internal needs or internal desires [14]. We define it procedurally as follows: An internal drive for each student, making them ready to learn with love and passion based on internal and external circumstances and beliefs that affect them and that drive their behavior to achieve the goals they deem important. This is measured by a special scale prepared by the researchers specifically for this purpose.

Non-formal Education. Ref. [2] defined non-formal education as continuous and organized educational activities of varying duration that do not meet the definition of organized education for people of different ages. It is possible that this education is implemented inside educational institutions or outside their usual authority to obtain something that would address educational requirements that are recognized and legitimate, according to certain facilities that mitigate the severity of strict regulations. We define it procedurally as follows: Educational programs that are carried out in a systematic, planned manner and that contain various educational activities taking place outside the regular educational system but under the umbrella of the MOE. These programs are characterized by flexibility and consideration of learners' conditions, characteristics, traits, capacities, and ages and work side by side with learners' formal education.

The Culture Promotion Program for Dropouts. This non-formal education program seeks to provide students (13- to 20-year-old females and 13- to 18-year-old males) who have dropped out of school with a set of skills, knowledge, and attitudes. It emphasizes their neglected educational rights and develops their professional maturity 
by retraining and qualifying them to join the Vocational Training Corporation, where the duration of the program is 24 months as a maximum [2]. We define it procedurally as a non-formal education program that is implemented in many centers. The program consists of three levels and covers the academic grades from the first grade to the tenth basic grade; it is based on a curriculum approved by the MOE. When students pass the third level, they obtain the tenth-grade basic certificate, which qualifies them to join home study or vocational training institutions according to their desire.

\subsection{Previous studies}

There were numerous studies dealing with the topic of blended learning and its impact on developing critical thinking skills because of the great importance of this topic in different societies. Next, we discuss the most prominent of the Arab and foreign studies that dealt with this topic.

Ref. [7] studied the effect of teaching physics using the blended learning model on motivation to learn and of acquiring scientific concepts among ninth grade students in Jordan. He prepared educational materials using a blended learning model for the experimental group while the control group studied in the usual way. The sample of the study consisted of 59 students $(n=29$ experimental, $n=30$ control) in the Directorate of Education of the Second Zarqa District for the 2014/2015 academic year. They were chosen by intentional method. Results revealed statistically significant differences between the students' performance scores on the scale of learning motivation and in the test of acquiring scientific concepts in favor of the experimental group.

Ref. [17] conducted a study aimed to improve critical thinking skills and students' motivation by implementing inquiry mind maps. The study used a quasi-experimental group with a non-equivalent control group pretest-posttest design. The experimental class used an inquiry mind map, whereas the control class required conventional learning. The sample consisted of 206 students from different schools and of different genders. Data on critical thinking skills was obtained from essay tests. Motivation data learning was taken from instrument motivation toward science learning. Data of critical thinking skills and learning motivation were analyzed using ANCOVA. Their findings showed that there were differences in students' ability to think critically and in their learning motivation when using an inquiry mind map tool. They found no differences between school and gender on critical thinking skills and learning motivation. The study results showed that the inquiry mind map tool had an impact on increasing critical thinking skills and learning motivation. It was recommended to develop other learning tools and a learning model to improve critical thinking skills.

Ref. [5] conducted a study on the effectiveness of the combination between the use of the smart blackboard and computer simulation in developing both internal motivation and metacognition skills, and scientific and technological skills of ninth grade students in Palestine, following a quasi-experimental approach. The household electricity unit with technology research was taught in the second semester of the 2014/2015 academic year; the study sample consisted of 82 students $(n=40$ experimental, $n=42$ control). Results showed statistically significant differences between the mean scores of the control and experimental groups in the post-application of the scientific skills assessment 
card and the metacognitive skills scale in favor of the experimental group, but none for either group in the post-application of the internal motivation scale.

Ref. [4] conducted a study aimed at investigating the effect of the blended teaching method on the achievement of fourth grade students in basic mathematics and on their motivation toward learning it in Jordan. It was conducted through the use of the quasiexperimental curriculum. The sample consisted of 71 students (male and female) from the model school of the University of Mu'tah for the 2006/2007 academic year. The sample included 38 students in the experimental group and 33 students in the control group, who were deliberately chosen. The study found statistically significant differences in post-academic achievement and the motivation scale in favor of the experimental group.

Another case study [18] aimed to identify the difficulties that affect motivation and attitude when using an e-learning system by 154 Jordanian university students. It used the Technology Acceptance Model to construct a model that tested the relationship among the difficulties using and attitudes toward e-learning systems through use of a synchronized e-learning system called BigBlueButton. The quantitative questionnaire data were analyzed using an AMOS tool to discover the direct and indirect paths between the study's variables. The significant results showed strong effects of students' motivation (usefulness and ease of use) on the relationship among three e-learning difficulties and their attitude toward the use of these systems.

Ref. [15] studied the effects of learning motivation, self-efficacy, and blended learning on Indonesian high school students' achievement in the Industrial Revolution 4.0. The study found that learning motivation and blended learning had a positive and significant effect, that self-efficacy had no significant effect, and that learning motivation, self-efficacy, and blended learning together had a significant effect on these students' achievement.

The aim of a qualitative study conducted by Ref. [11] was to study three psychosocial impacts (i.e., building resilience and combating posttraumatic stress disorder, capacity building and peace strategies, information security) of the Ideas Box on society in the East African Republic of Burundi. The study targeted those under the age of 30 who were divided into proportional age groups for conducting focus sessions or interviews. Multiple tools were used; more than 100 individual or group interviews were conducted, targeting 142 in focus sessions consisting of 7-11 persons in each group, in addition to observation. Results showed a noticeable impact and improvement in the psychosocial effects of the Ideas Box on society, especially in relation to asylum.

The case study conducted by Ref. [10] to study the trends of Ideas Box users during the first six months of use in the Mosasa and Kafumo camps in the East African Republic of Burundi, in particular, the early effects on enhancing the quality of education, protecting the child, and strengthening community ties. The study found early positive trends toward the Ideas Box, which helped in a strong increase in registration and diversity of users. This was confirmed by qualitative assessments that showed a strong influence of the apparatus on establishing community ties within the camps. Although adults were more likely to sign up for the Ideas Box, children and adolescents used the box frequently and regularly. This highlights the outstanding contributions of the Ideas Box to education, either through school activities or support outside school hours. There 
was a consensus that the Ideas Box is a safe space that helps reduce youth exposure to external hazards. Despite these initial positive effects, they identified many challenges, especially with regard to women's participation in activities and the need for extensive, long-term studies to determine the impact of the program on academic performance, the psychological and social environment, and the openness of refugees.

\section{$4 \quad$ Method and Procedures}

This section describes the methodology of the study, the study community and its sample, the study tool and how it was developed, the procedures used to verify the validity and stability of the tool, and the statistical methods used in processing data related to the response of the sample members to the study questions and study procedures.

\subsection{Study methodology}

This study used the quasi-experimental approach to achieve its goals. The JOHUD Center for Social Support affiliated to the Jordanian Hashemite Fund was chosen intentionally, while the people inside the school were randomly selected. The reasoning is to have one as a control group, and another experimental group of non-formal education female students. Blended learning was implemented using the Ideas Box with the experimental group, and the normal method was applied to the control group.

\subsection{Study sample}

The sample of the study consisted of 30 female students of non-formal education in the Culture Promotion Program for Dropouts at the Center, who were in two groups. The participants (14-18 years old) had dropped out for three or more years and cannot go back to formal schools according to MOE regulations. There are various reasons for not attending school, including economic, social, religious, and cultural reasons. The sample was chosen by a systematic random method during the second semester of $2019 / 2020$.

\subsection{Study tools}

The two researchers prepared an instructional program based on blended learning using the Ideas Box. It consisted of tailored lessons and various resources on the Integrative Approach (Unit 1 - Pillars of Islam) and lasted for six weeks. We presented the teaching program to a group of experts (e.g., supervisors and education specialists) who evaluated it; we then made modifications based on their feedback.

The teaching program is based on active learning methods; and the program is basically a set of specific and structured steps that are based on the techniques, pedagogical and educational methods. The experimental group was taught the Pillars of Islam unit, using active learning methods for one hour daily. It combined the use of regular learning 
and its various active strategies with the activation of blended learning using the Ideas Box. It included a set of information, experiences, structured exercises, activities, and skills that motivates school dropouts to learn and help them keep pace with the new developments. This will also help satisfy their different needs as well as achieve fun and self-learning at the same time.

\subsection{Motivation toward learning}

The building of the learning motivation scale was based on Goal Theory, which is one of the most recent theories that explains learning motivation and its importance. It assumes that individuals are more motivated when they have an orientation toward learning goals (mastery). Scholars of this theory (e.g., Dweck, Meece, Ames) have shown that the learning goals have a great relationship with the emotional side of the student. Goals are positively related to pride and satisfaction in the case of success and negatively related to anxiety in the case of failure. Scholars argue that students' learning motivation is affected by their social and family environment, school factors, and personal factors. To verify the validity of the scale of the students' motivation toward their learning, we used the validity of the peer reviews of specialists at the University of Jordan, workers in the humanitarian field, and specialists in international organizations and national institutions. To verify the stability of the scale, it was applied on a pilot sample. The test was repeated and the time interval between the first and second application was two weeks. The internal consistency method Cronbach alpha was also used, where the results showed a value of 0.861 . The stability of the repetition was calculated, reaching 0.94 .

\section{$5 \quad$ Results}

The data were analyzed using analysis of variance (ANCOVA). The statistical treatment was done through the use of the SPSS statistical analysis program. Findings related to the study question-Are there statistically significant differences $(0.05 \geq \alpha)$ between the mean scores of the study subjects in the motivation of the students toward learning due to the method of teaching using blended learning based on the Ideas Box?-are presented next.

To answer the question, ANCOVA and the extraction of the arithmetic means and standard deviations of the experimental and control groups were used in the pretests and posttests for the motivation of the students toward learning. They are attributed to the blended learning based on the Ideas Box. Table 1 shows the apparent differences between the averages of the experimental and control groups on the scale of motivation to learn. The arithmetic mean of the premeasurement of the experimental group reached 3.4593 , and the postmeasurement of the experimental group increased to 3.8833. However, for the control group, the arithmetic mean of the premeasurement reached 3.7611, and the postmeasurement of the control group rose to 3.9889 . 
Table 1. Arithmetic averages and standard deviations of the two experimental and control groups for the pretests and posttests of motivation

\begin{tabular}{|l|l|c|c|}
\hline \multicolumn{2}{|c|}{ Group } & \multicolumn{2}{c|}{ Motivation } \\
\hline \multirow{2}{*}{ Experimental } & Arithmetic mean & 3.4593 & Posttest \\
\cline { 2 - 4 } & Standard deviation & .39169 & .3833 \\
\hline \multirow{2}{*}{ Control } & Arithmetic mean & 3.7611 & 3.9889 \\
\cline { 2 - 4 } & Standard deviation & .27334 & .24461 \\
\hline \multirow{2}{*}{ Total } & Arithmetic mean & 3.6102 & 3.9361 \\
\cline { 2 - 4 } & Standard deviation & .36565 & .27635 \\
\hline
\end{tabular}

To know whether these differences were statistically significant or not, the ANCOVA was used. Table 2 shows that there are no statistically significant differences at the level of significance $(\alpha \leq 0.05)$ between the averages of the two groups: the experimental and control groups in the measure of motivation toward learning, where the value of $F=0.832$. The statistical significance reached 370 , and the size of the effect of the independent variable on the dependent reached 0.030 . The adjusted arithmetic mean of the experimental group (3.885) was less than that of the control group (3.988).

Table 2. ANCOVA results for differences between the two groups for motivation test

\begin{tabular}{|l|c|c|c|c|c|c|}
\hline \multicolumn{1}{|c|}{ Source } & Total squares & $\begin{array}{c}\text { Degrees of } \\
\text { freedom }\end{array}$ & $\begin{array}{c}\text { Average of } \\
\text { squares }\end{array}$ & F value & $\begin{array}{c}\text { Statistical } \\
\text { value }\end{array}$ & Aita value \\
\hline Group & .066 & 1 & .066 & .832 & .370 & .030 \\
\hline Pre motivation & .000 & 1 & .000 & .003 & .959 & .000 \\
\hline Error & 2.131 & 27 & .079 & & & \\
\hline Total & 2.215 & 29 & & & & \\
\hline
\end{tabular}

\section{Discussion of Results}

The results indicated that there were no statistically significant differences at the level of significance $(\alpha \leq 0.05)$ between the averages of the two groups, experimental and control, in the measure of motivation toward learning, where the value of $F=0.832$ and the effect of the independent variable on the dependent reached 0.030 . This result proves that there were no statistically significant differences despite what the Ideas Box provides in terms of various media, including sound, image, and movement, or through the available library while using a new method that is not traditional but one that suits and copes with the era in which the study participants live and in the language they understand. Although the designed program attempted to focus on strengthening social relations and human aspects between the student and the teacher and among the students themselves, there were no statistically significant differences. This may be attributed to the psychological and social conditions of these dropout female students. They do not live-in easy circumstances and require psychological and behavioral intervention in certain cases. Supportive service is provided by the counseling specialist at the JOHUD Center, and it may require varying periods depending on the case. 
We note that a sense of safety is one of the most important causes of psychological stability for students, which helps them enhance their motivation. This is done through focus groups that the researcher held. Focus groups are within the researcher's field of work. Prior to the study; she has conducted these groups with other students who were in their last semester of school and who did not participate in this study. The female students expressed their conflicting feelings before joining the Center and at every stage, right up to graduation. Some felt safe after a year, and some did not. The history of each student plays a big role in explaining this in terms of the nature of social life, the family's confidence in the importance of the role of girls in life, and their ability to make decisions that concern them, even if at a simple level.

Also, the student's awareness of the importance of her presence in life, her self-esteem and her role, has a major role in the extent of her motivation to learn in general. In most cases of dropout students, the main reason for the dropout was their parents' lack of confidence in their ability to cope with the challenges they would face alone, as well as their inability to confront and avoid harassment. In the Arab culture, the male role is key in protecting females; girls must adhere to their decisions without discussion, which negatively affects their self-confidence, or their confidence that any work they do will have a positive result later. At any time, in some families, males decide not to send female students to the Center for reasons that are convincing from their point of view.

On the other hand, customs and traditions in some families (e.g., early marriage and that the destination of every girl is her home) have contributed to these girls dropping out. In many cases of dropout, we found that girls had experienced either a previous engagement, as represented in a marriage contract, or a previous marriage that ended in divorce. In rare cases of early marriage did the husband agree to allow his wife to complete her education. When looking at individual cases, we noted that each girl faced a large number of psychological disorders and challenges, resulting in a sense of inferiority in some. Dealing with and/or getting out of all these exhausting situations takes a long time, effort, continuity and follow-up, and awareness of the parents and the student herself.

In normal circumstances and according to human nature, and in the case of regular students who did not go through similar conditions as the dropout students, it takes time for students to build bridges of confidence and feel it in a new place that they join. When we reflect this on the dropout female students, building trust takes much longer. In many cases it is necessary, first, to overcome and solve previous problems and, second, to modify behaviors before reaching the confidence-building stage. Accordingly, our results may be attributed to the short period of the program designed using the Ideas Box. On the other hand, female students need time to build effective and fruitful social relationships with their colleagues, as well as with the facilitator and the Center's employees. Therefore, one of the most important measures that the Center is taking besides the educational side is providing interesting and enjoyable activities related to life skills, psychosocial support, and recreational activities that allow them to integrate in the Center and with those inside it including students, facilitators, and employees.

This result may be attributed to the female students' lack of self-confidence regarding the technology represented by the Ideas Box, because of their recent enrollment in 
the Center, and the large size of the box and its equipment and tools with which they have no prior experience. They have never seen it before; and, in most cases, they do not possess basic technological skills, which sometimes creates frustration about the possibility of success in using the Ideas Box. This feeling might also happen when they work in groups and see that some peers have the ability to use it.

The results of this study disagrees with those of Ref. [7], which aimed to know the effect of teaching physics using the blended learning model on motivation to learn and the acquisition of scientific concepts among ninth grade students. The results of the present study revealed a statistically significant difference between students' attainment scores on the scale of learning motivation attributable for the method of teaching and for the benefit of the experimental group that studied the blended learning model. It is consistent with the results of Ref. [5], which aimed to study the effectiveness of the combination between the use of a smart board and computer simulation in developing internal motivation, metacognition skills, and practical skills in technology among ninth grade students in Palestine. It showed no statistically significant differences between the two averages of the scores of the control and experimental groups in the postapplication of the internal motivation scale.

The results of this study also disagree with those of Ref. [11], which aimed to study three psychosocial effects of the Ideas Box on society in the East African Republic of Burundi. There it showed a significant impact and improvement in the psychosocial effects of the Ideas Box on society, especially in case of asylum. It also disagreed with the outcomes of a case study conducted by Ref. [10], which examined the trends of Ideas Box users during the first six months of use in the Mosasa and Kafumo camps in the East African Republic of Burundi. In order to know the early effects on enhancing the quality of education, protecting the child, and strengthening community ties, the study found early positive trends toward the Ideas Box, which helped in a strong growth in registration and diversity of users. This is confirmed by the qualitative evaluations that were conducted, which showed a strong influence of the apparatus on establishing community ties within the camps. It also disagreed with the study of Ref. [4], which aimed to investigate the impact of the blended education method on the achievement of students in the fourth grade in basic mathematics and their motivation toward learning it. The results indicated that there is a statistically significant difference between the mean scores of the experimental group students on the motivation scale and in favor of the experimental group.

\section{$7 \quad$ Recommendations}

Based on the results of the present study, we recommend the following:

- Reconsider the design of the Culture Promotion Program for Dropouts so as to include blended learning in general, and the Ideas Box in particular, in a systematic, organized, and thoughtful manner.

- Conduct more research studies on the impact of blended learning in general, and the Ideas Box in particular, in increasing students' motivation. 


\section{References}

[1] Aldershwi, A. A. (2019). The effect of the blended learning strategy on academic achievement and information retention of eleventh grade literary students in the geography subject in Duhok Governorate / Iraq. Educational Sciences Studies, 46(1), 271-286.

[2] Al-Nasser, A. (2013). Non-formal education in Jordan. Amman, Jordan: Ministry of Education.

[3] Al-Sayed, A. (2002). Teaching technology and instructional aids ( $1^{\text {st }}$ ed.). Cairo, Egypt: Arab Thought House.

[4] Al-Zoubi, A., \& BaniDomi, H. A. (2012). The impact of blended teaching on the achievement of the fourth-grade students in mathematics and their motivation towards learning. Journal of the University of Damascus, 28(1), 485-518.

[5] Ayyad, F. I., \& Al-Nahhal, A. N. (2018). The effectiveness of combining the use of a smart board and computer simulation in the development of internal motivation and metacognition skills and practical skills in technology among ninth grade students in Palestine. Educational Sciences Studies, 45(2), 231-255.

[6] CARE International, Plan International. (2018). My vision for a safe and equal future: An adolescent and youth-focused assessment on gender-based violence and sexual and reproductive health and rights in Jordan.

[7] E'limat, A. (2018). The effect of teaching physics using the blended learning model on motivation to learn and acquire scientific concepts among ninth grade students. Educational Sciences Studies, 45(2), 51-67.

[8] Heinze, A., \& Procter, C. (2004). Reflections on the use of blended learning. Proceedings of Education in a Changing Environment.

[9] Libraries Without Borders. (n.d.). Ideas Box. https://www.librarieswithoutborders.org/ideasbox/

[10] Libraries Without Borders. (2014). Ideas Box Burundi: Midterm report-Initial results and perspectives. https://www.bibliosansfrontieres.org/wp-content/uploads/2017/08/BSF Report-IDB-Initial-Results-Burundi-2015-ENG.pdf

[11] Libraries Without Borders. (2015). Ideas Box: An innovating psychosocial tool for emergency situations - Impact study in the Kavumu and Bwagirisa camps, Burundi. https://www.bibliosansfrontieres.org/wp-content/uploads/2017/08/BSF Report-IDBPsychosocial-Impact-2015-ENG.pdf

[12] Ministry of Education. (2018). Education strategic plan for years 2018-2022. Amman, Jordan: Author.

[13] National Centre for Human Resources Development. (2016). Education for prosperity: Delivering results (A National Strategy for Human Resource Development 2016-2025). Amman, Jordan: Author.

[14] Qatami, Y., \& Adas, A. (2002). General psychology. Amman, Jordan: Dar Al-Fikr for Printing \& Publishing.

[15] Rafiola, R., Setyosari, P., Radjah, C. \& Ramli, M. (2020). The effect of learning motivation, self-efficacy, and blended learning on students' achievement in the Industrial Revolution 4.0. International Journal of Emerging Technologies in Learning (iJET), 15(8), 71-82. https://doi.org/10.3991/ijet.v15i08.12525

[16] Salem, H. (2018). The voices of reason: Learning from Syrian refugee students in Jordan. Policy Paper No. 18/3. REAL Centre, University of Cambridge. https://doi.org/10.5281/zenodo. 1247330 
[17] Sari, R., Sumarmi, S., Astina, I., Utomo, D., \& Ridhwan, R. (2021). Increasing students critical thinking skills and learning motivation using inquiry mind map. International Journal of Emerging Technologies in Learning (iJET), 16(3), 4-19. https://doi.org/10.3991 /ijet.v16i03.16515

[18] Shakah, G., Al-Oqaily, A. T., \& Alqudah, F. (2019). Motivation path between the difficulties and attitudes of using the E-Learning systems in the Jordanian Universities: Aajloun University as a case study. International Journal of Emerging Technologies in Learning, 14(19), 26-48. https://doi.org/10.3991/ijet.v14i19.10551

\section{Authors}

Asma'a Alkhatib is an early childhood and adolescent education expert working in Jordan in the humanitarian sector since 2006. She is a technical advisor in early childhood education (formal, informal, non-formal) in both camps and host communities with Jordanians and refugees. She also works as a senior project manager in the Early Childhood Care and Development (ECCD) and Education project at Plan International in Jordan and represents her organization in the ECCD and Education working group nationally and globally.

Suhair Jaradat is an Associate Professor of Educational Technology in the Department of Curricula and Instruction at the University of Jordan. She earned her PhD in Educational Technology from the University of Missouri-St. Louis, USA, in 2004. She has published several research papers on integrating technology in the classroom, online teaching and learning, cognition and technology, and education for sustainability. Email: s.jaradat@ju.edu.jo.

Article submitted 2021-02-11. Resubmitted 2021-03-10. Final acceptance 2021-03-10. Final version published as submitted by the authors. 